\title{
A method of data transmission technology for line condition monitoring in smart transmission grid
}

\author{
Congcong $\mathrm{Li}^{1}{ }^{1,}$, Qing Wang ${ }^{1}$, Zhen Jing ${ }^{1}$, Hongxia Zhu ${ }^{1}$, Pingxin Wang ${ }^{1}$ and Chao $\mathrm{Yu}^{1}$ \\ ${ }^{1}$ State Grid Shandong electric power company marketing service center (Metering Center)
}

\begin{abstract}
In order to make the operation of the line more safe and reliable, the state monitoring is needed and the remote transmission of data is realized on this basis. This paper proposes a variety of communication modes suitable for transmission line condition monitoring data transmission. The corresponding communication system design is given for different application conditions. The development and application of data transmission technology for condition monitoring of transmission lines are discussed in combination with two different schemes of typical design and distributed intelligent fault diagnosis system.
\end{abstract}

\section{Introduction}

The construction of smart grid is to achieve the overall strategic planning goal of power development ${ }^{[1-2]}$. Therefore, it is necessary to build the line condition monitoring system, adopt more new technologies, use more reliable communication methods, realize the realtime data transmission, speed up the transmission speed, reduce the communication cost, improve the management and control level of transmission line, and deal with emergencies in time. The research on related technologies started late in China. In the early 21 st century, China began to carry out regular inspection and maintenance of power supply departments and power plants and other facilities and equipment. However, the initial maintenance methods are determined by referring to the previous traditional experience, and the implementation technology is poor and relatively rigid ${ }^{[3-}$ 5]. With the continuous development of sensor equipment, communication network, computer technology and other disciplines, the current transmission line has high technical support in data storage, remote measurement, real-time response and other aspects. It makes the transmission line online monitoring technology level achieve better development, so that the data long-distance transmission technology can be realized.

\section{Condition monitoring system}

\subsection{Architecture system}

The framework of the monitoring system is shown in Figure 1, which is composed of three parts. First, the master station, including access network shutdown $(\mathrm{CAG})$, database services and other functional modules.
It can monitor all kinds of equipment and reduce the difficulty of data storage, management and application ${ }^{[6]}$. CMG is a kind of gateway equipment, which is divided into two different types: line and substation. It can remotely connect with the condition monitoring agent, obtain the condition monitoring data and verify it, so as to realize the reasonable control of data information. Second, condition monitoring agent (CMA) can realize the management and cooperation of lines in local area, collect all kinds of measurement data and communicate with master station. It can be connected to various types of condition monitoring devices, which is more intelligent and safe. Third, the condition monitoring device (CMD), which is installed on the monitored equipment, has multiple functions such as data management and communication, and can interact with other modules to transmit control instructions to the data acquisition unit. CAG is similar to gateway in function. It is located at the edge of the master station and can be connected with the master station through CMA. CMA acts as a bridge, which is connected with CMD and CAG through the interface, and is mostly set at the substation; CMD is the end node, which is set on the tower. As shown in Figure 1.

\subsection{Construction target}

The main monitoring station and terminal should meet the following requirements in the construction process. First of all, the construction of the main station. It needs to receive, store and analyze the terminal data; monitor the current and historical load of the line, and make statistics, so as to ensure that the line can have referential data information in the process of transformation. It realize the location of various types of fault section, so as to shorten the time when finding the fault. Analyze

\footnotetext{
* Corresponding author: shulin1023@sina.com
} 
the fault and recording information, so as to find the root cause

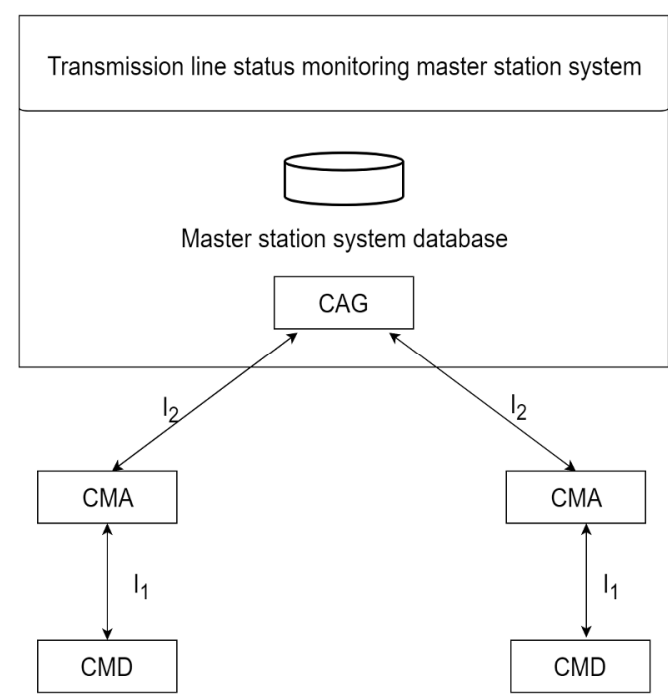

Figure 1. Transmission line condition monitoring overall block diagram of communication system

and evolution of the fault as soon as possible ${ }^{[8]}$. It must have the function of query and statistics of historical faults, and provide technical support; through the forward and reverse isolation device docking with the master station, it can realize the rapid fault line selection and positioning, and connect with the master station, so as to ensure that the master station can obtain the transmission information in a short time. Secondly, the goal of terminal construction. It must meet the installation requirements of a variety of wire diameter, and monitor the line load, field strength and other parameters. The real-time monitoring terminal status and the application of GPRS technology promote the line status report more timely and reliable. It realize solar power supply and support backup power supply.

\section{Data transmission technology}

\subsection{CMA technical solution}

At present, there is no simple communication equipment in the transmission line condition monitoring system, mainly through CMA equipment networking for communication. CMA equipment is the main equipment in the communication network. It is equipped with multiple interface communication modules, which can not only realize the additional processing of data, but also realize the multi-directional communication of data. CMA and CAG are connected through 12 interface communication protocol. Because CMA and CAG are far away from each other, they need many and complex communication networks. Therefore, the hybrid networking mode is selected to combine all kinds of private network communication modes and put into use. The hybrid networking mode of any two of Ethernet optical fiber communication module, passive optical network optical fiber communication module and wireless private network communication module can be selected. CMA and CMD are connected by 11 interface communication protocol, and the distance is relatively close. The network modules can be selected, including Ethernet electric communication module, wireless private network communication module, serial port module, etc.

From the above analysis, the full-function CMA must include several modules: CMA core processing module, security encryption module, power supply module, 11 interface module and 12 interface module, among which 11 interface module is composed of Ethernet electrical communication module, wireless private network communication module and serial port module The interface module consists of Ethernet optical fiber communication module, wireless public network communication module and OLT_PON communication module, wireless private network communication module and ONU_ PON communication module consists of five parts. Full function CMA has many kinds of modules, high cost and relatively high power consumption, so it will be appropriately cut and serialized when used in the actual transmission network to avoid waste of resources as much as possible, and only equipped with relatively necessary communication modules.

\subsection{Typical design scheme}

1) The CMD is located on the tower with optical cable junction box

A CMA should be installed on the tower to communicate with CAG by means of optical fiber communication module. For the communication mode above optical fiber communication module, it is often selected from passive optical network optical fiber communication module and Ethernet optical fiber communication module.

2) The CMD is located on the tower without optical cable junction box

The simplest way is to configure the AC mode of the wireless private network communication module in the CMD system, and communicate with the adjacent CMA (configured as AP mode) with the help of the wireless private network communication module. When CMA is on the tower with optical cable junction box, the communication between CMD and CMA is realized by 11 interface and upward communication is realized by 12 interface. For the communication mode above optical fiber communication module, the best choice is passive optical network optical fiber communication and Ethernet optical fiber communication. When CMA is far away from the tower with optical cable junction box, or even the direct communication is obstructed, the wireless relay mode of wireless private network communication module can be selected to complete the communication. Among them, CMA0 and CMD use the 11 interface wireless private network communication module to realize the communication function, while cmal and CMA0 use the 12 interface wireless private network 
communication module to realize the communication function.

At this time, the uplink communication of CMA mainly refers to the communication between CMA0 and cmal with the help of wireless private network communication module, which puts forward higher requirements for CMA0 and must have the relay ability of wireless private network communication module. In wireless private network communication mode, cmal is AP mode, cma0 is relay mode, and CMD is AC mode.

With the help of the wireless mode, the communication channel is set up with the lines with OPGW cable resources, and the system access of the CMD module is completed through the OPGW resources, or through the wireless public network communication mode.

\section{Intelligent grid control}

At present, due to the different needs and considerations of smart grid in different countries according to their own national conditions, there is no unified and clear definition of smart grid. In the United States, the proposal of smart grid originates from the gap in power supply reliability of traditional power grid and the demand for information industry. Therefore, its smart grid emphasizes the application of information technology. European countries and Japan mainly put forward smart grid from the perspective of renewable energy development demand, which is characterized by the compatibility of power grid with renewable energy generation. According to the law of power grid development, China puts forward the concept of strong smart grid, highlighting the characteristics of cross regional optimal allocation of resources and diversified utilization of energy. In a broad sense, smart grid includes intelligent dispatching system that can give priority to renewable energy, intelligent metering system that can dynamically price, and intelligent technology system that can optimize load balance by adjusting the power of power generation and electrical equipment. Electric energy not only flows from the centralized power plant to the transmission network, distribution network and users, but also has various forms of new energy and energy storage equipment, such as solar energy, wind energy, fuel cells, electric vehicles, superconducting energy storage, etc. In addition, the high-speed, two-way communication system realizes the information interaction between the control center and the power grid equipment, and guarantees the advanced analysis tools and decision-making system. The security, stability and optimal operation of smart grid are discussed. One of the core contents of smart grid control is to analyze the dynamic behavior of power system under disturbance, determine the appropriate countermeasures, prevent the occurrence of accidents or avoid the expansion of accidents. The development of information and communication, intelligent analysis technology and power electronics technology promotes the progress of smart grid control decision-making technology and the improvement of control ability.
Building a powerful and highly integrated information system is the foundation of smart grid. The high-speed, two-way, real-time and integrated communication system makes smart grid a large-scale infrastructure for dynamic, real-time information and power exchange interaction. When the communication system is completed, the smart grid realizes its selfhealing characteristics through continuous selfmonitoring and correction, and the application of advanced information technology. The information system can also monitor various disturbances and redistribute the power flow to avoid the expansion of the accident. The high-speed two-way communication system enables various intelligent electronic devices (IEDs), intelligent meters, control centers, power electronic devices and their protection systems, as well as users to carry out network communication, so as to improve the control ability and service level of the power grid. For communication technology, we need to focus on the development of two aspects of Technology: open communication architecture, so as to form a "plug and play" environment, to ensure that the network communication between power grid components can be carried out. Pen communication architecture Unified technical standards enable all sensors, intelligent electronic devices and application systems to achieve seamless communication, that is, information can be fully understood between all these devices and systems, and realize the interoperability between devices, devices and systems, systems and systems.

Optical fiber communication technology and wireless communication technology constitute the basic communication mode of smart grid in the future. Power line carrier and power special optical cable will be applied to a certain extent. Multiplexing technology, long-distance transmission technology, automatic switched optical network technology, packet transport network technology and so on in optical fiber communication technology will become the important technology development direction in this field. Traditional digital microwave communication will also be the main communication mode of power system. In addition, satellite communication and mobile broadband communication will also be applied in the field of business support. Parameter measurement technology is the perception link of information system and the basic component to realize the control function of smart grid. Advanced parameter measurement technology obtains data and converts it into data information for all aspects of smart grid.

Through parameter measurement, we can evaluate the health status of power grid equipment and the integrity of power grid, read meters, eliminate the estimation of electricity charges, manage power consumption, reduce power grid congestion and realize communication with users. The smart meter can realize the measurement and control of user related parameters. Through the phasor measurement unit (PMU), wide area measurement system (WAMS), component dynamic monitoring, various advanced sensors and communication technology, the system can achieve rapid 
simulation, intelligent pre control, intelligent recovery and other functions.

As the latest achievements of modern information technology, spatial information technology and stream media technology will play an important role in the information processing of smart grid. Spatial information technology includes geographic information system (GIS), remote sensing technology and global positioning system (GPS). Streaming media technology refers to the continuous time-based media using streaming transmission technology in the Internet. In the application of information technology, the combination of knowledge acquisition and data mining, data warehouse and online analytical processing will constitute the key content and technical difficulties of information processing in smart grid.

Power system simulation is to establish physical or mathematical model according to the actual power system, carry out calculation and test, and study the working behavior and characteristics of power system in the specified time. Power system simulation plays an important role in power system planning, design, operation, test and training. In the environment of smart grid, HVDC, facts, security and stability devices are applied to power system, and the simulation problem presents the requirements of multi time scale, strong nonlinearity and high precision. Some new simulation algorithms and platforms are emerging. Electromechanical transient electromagnetic transient hybrid simulation technology can reasonably simulate the fast process of power electronic equipment and the slow process of traditional electromechanical equipment. In parallel computing, the large-scale power system is divided into several sub networks. Different sub networks are used for parallel computing, and reasonable communication data flow is maintained among the sub networks, so as to realize real-time or even super realtime computing of large-scale power grid. With the development of the requirements of smart grid for simulation technology, the research of fast simulation algorithm, basic simulation data, simulation model, large-scale power system digital real-time simulation, and power grid visualization technology are still hot issues in the research of simulation technology.

On the one hand, smart grid is the product of electrical technology, new energy technology and information technology, on the other hand, it puts forward new challenges to these technical fields. Smart grid embodies the vision of the future power grid development, and the realization of its established goal mainly depends on technological progress. Therefore, the smart grid control technology system integrates advanced equipment manufacturing technology, information and communication technology, standard and test evaluation technology and many other technologies. Among them, information and communication technology is the "central nerve" to realize the control function of smart grid, and power electronics and energy storage technology act as the "executive agency" of smart grid control, Standards and test evaluation constitute the system and management guarantee for the smooth implementation of smart grid control.

\section{Conclusion}

The transmission technology of intelligent transmission network line status monitoring data should be adjusted in combination with the system construction requirements. It achieve the purpose of data security, stable transmission and optimization of system architecture. So that it provide strong technical support for intelligent monitoring of power system such as fault diagnosis, pole tower tilt, dancing, ice coating, insulator pollution removal and arrester. It effectively ensures the safety, health and good of the line run smoothly.

\section{References}

1. Vedavalli K, Muruganantham N. Defended data transmission scheme based reliable metering for smart grid applications[J]. Cluster Computing, 2018.

2. Transmission of frequency balance instructions and secure data sharing based on chaos encryption in smart grid-based energy systems applications[J]. IEEE Access, 2021, PP(99):1-1.

3. Gao J, Zhang X, Hao L, et al. Joint encryption and compressed sensing in smart grid data transmission[J]. IEEE, 2014

4. Noelia Uribe-Pérez a, B I A, B D D L V, et al. Onfield evaluation of the performance of IP-based data transmission over narrowband PLC for smart grid applications - ScienceDirect[J]. International Journal of Electrical Power \& Energy Systems, 2018, 100:350-364.

5. Miyagawa S, Yamada T, Ninagawa C. Generic and realistic spatial deployment modeling on data transmission performance evaluation of multi-hop smart grid metering system[J]. Ieej Transactions on Electrical \& Electronic Engineering, 2017, 12(3):397-403.

6. An Approximately Optimal Algorithm for Scheduling Phasor Data Transmissions in Smart Grid Networks. IEEE Transactions on Smart Grid, 2015.

7. L Vrbský. Clustering-driven equipment deployment planner and analyzer for wireless non-mobile networks applied to smart grid scenarios. 2018.

8. Rahman M S. Optimizing Smart Grid Aggregators and Measuring Degree of Privacy in a Distributed Trust Based Anonymous Aggregation System[J]. 2020. 